\title{
Circular RNA hsa_circ_0069117 suppresses proliferation and migration of osteosarcoma cells lines via miR-875-3p/PF4V1 axis
}

\author{
Ziyan Zhang, Lin Zhou, Shicheng Zhou and Xin Li* (0)
}

\begin{abstract}
Background: Osteosarcoma (OS) is one of the most common malignant bone tumors in children and adolescents. Circular RNAs (circRNAs) are critical regulators involved in multiple physiological and pathological processes. However, the underlying regulatory mechanisms of circRNA in OS are still not fully understood.

Methods: The circRNA expression profiles were downloaded from the Gene Expression Omnibus (GEO) database and analyzed by GEO2R. Bioinformatics analysis was performed to predict the potential target miRNAs of hsa_ circ_0069117 and its downstream mRNAs. The co-expression of hsa_circ_0069117/miR-875-3p/PF4V1 axis was further validated in OS tissue samples via quantitative real-time PCR (qRT-PCR). Luciferase reporter gene plasmids containing the sequence of PF4V1 and hsa_circ_0069117 were constructed to verify the putative sites of miR-875-3p. Gain/ loss-of-function assays were performed to verify the effect of hsa_circ_0069117 on miR-875-3p/PF4V1 expression and related pathways via qRT-PCR and Western blot. Cell counting kit-8 (CCK-8) and wound-healing assays were performed to evaluate the effect of hsa_circ_0069117 on cell proliferation and migration of MG63 and U2OS, respectively.

Results: We identified hsa_circ_0069117 as the most markedly dysregulated circRNA in OS cell lines. Bioinformatics analysis indicated that hsa_circ_0069117 might inhibit the expression of miR-875-3p, thereby promoting the expression of platelet factor 4 variant 1 (PF4V1). The expression of miR-875-3p was negatively correlated to hsa_circ_0069117 and PF4V1 in clinical samples. Luciferase reporter gene assays confirmed the binding sites of miR-875-3p on hsa_circ_0069117 and PF4V1. Gain/loss-of-function and rescue assays further indicated that hsa_ circ_0069117 could significantly promote the expression of PF4V1 by sponging miR-875-3p, thereby inhibiting the proliferation and migration of OS cells by suppressing ERK1 and AKT.
\end{abstract}

Conclusion: Our study revealed that hsa_circ_0069117 is an anti-OS molecule that could substantially attenuate cell proliferation and migration of OS, which may provide a novel and reliable molecular target for the treatment of OS patients.

Keywords: Osteosarcoma, hsa_circ_0069117, miR-875-3p, PF4V1, Cell proliferation and migration

\section{Introduction}

Osteosarcoma (OS) is the most common primary malignant bone tumor accounting for $10 \%$ of solid tumors in children and adolescents $[1,2]$. It has been characterized

${ }^{*}$ Correspondence: lixin27@jlu.edu.cn

Orthopedic Medical Center, the Second Hospital of Jilin University,

Ziqiang Street 218, Changchun 130041, Jilin, China as a highly fatal disease with strong invasiveness and a high metastasis rate [2]. Aggressive chemotherapy plus surgery is the primary therapeutic method for OS with an overall 5-year survival rate of 70-80\% [3-5]. However, patients with lung metastases still have a low 5 -year survival rate of $5-10 \%$ and a high recurrent rate of $20-30 \%$ [3]. Although recent decades have witnessed significant progress in molecular targeting therapy of OS $[6,7]$, few original author(s) and the source, provide a link to the Creative Commons licence, and indicate if changes were made. The images or other third party material in this article are included in the article's Creative Commons licence, unless indicated otherwise in a credit line to the material. If material is not included in the article's Creative Commons licence and your intended use is not permitted by statutory regulation or exceeds the permitted use, you will need to obtain permission directly from the copyright holder. To view a copy of this licence, visit http://creativecommons.org/licenses/by/4.0/. The Creative Commons Public Domain Dedication waiver (http://creativeco mmons.org/publicdomain/zero/1.0/) applies to the data made available in this article, unless otherwise stated in a credit line to the data. 
advances have been made in promoting survival rate during the last 25 years, especially for metastatic disease [3]. Thus, further insight into the regulation mechanism and new therapeutic approaches of OS are urgently needed to address this problem.

Circular RNAs (circRNAs) were discovered decades ago $[8,9]$. It is a type of ncRNA (non-coding RNA) that has been suggested to act as an essential biomolecule in regulating multiple progression of diseases [10,11]. Circular RNAs have a unique feature to resist most RNases, enabling them to be ideal biomarker for detecting human diseases $[9,12]$. It has been proven that circRNAs can bind miRNAs and proteins thereby regulating multiple physiological and pathological processes [13]. Preliminary studies have revealed that some circRNAs are dysregulated in OS, resulting in changes in proliferation, invasion, migration, metastasis, apoptosis, adhesion and multi-drug resistance of OS cells $[14,15]$.

In the present study, we analyzed the GEO dataset GSE96964 and found that circRNA hsa_circ_0069117, a circular transcription of TBC1 domain family member 14 (TBC1D14), was most markedly dysregulated in OS cells [16]. However, the regulatory effect of hsa_circ_0069117 remains unclear. We validated its expression in OS cell lines and tissues, and further demonstrated that hsa circ_0069117 could significantly promote the expression of PF4V1 by sponging miR-875-3p, thereby promoting the expression of cell proliferation and migration of OS by upregulating extracellular signal-regulated kinase 1 (ERK1) and protein kinase B (AKT).

\section{Materials and methods}

\section{Differentially expression analysis of circRNA and miRNA} in OS

The circRNA and miRNA expression profiles of OS were downloaded from the Gene Expression Omnibus (GEO) database of the National Center of Biotechnology Information (NCBI, http://www.ncbi.nlm.nih.gov/geo/). The raw data of circRNA and miRNA expression profiles were then analyzed via GEO2R (https://www.ncbi.nlm. nih.gov/geo/geo2r/), an interactive web tool that allows users to identify genes or ncRNAs expressed differentially across experimental conditions $[16,17]$. The differentially expressed miRNAs were screened based on criteria of $\mid$ fold change (FC) $\mid \geq 2$ and adjusted $p<0.05$.

\section{Target prediction}

The target miRNAs of hsa_circ_0069117 were predicted using circBank (http://www.circbank.cn/searc hCirc.html). The Functional Enrichment analysis tool (FUNRICH) was used to screen out the potential target mRNAs of miR-875-3p in miRDB, TargetScan, miRWalk, TargetMiner, and microT-CDS databases [18].

\section{Cell culture}

Human osteosarcoma cell lines (OSCL) U2OS, 143B, MG63, HOS, and human mesenchymal stem cell line hMSCs were obtained from the American Type Culture Collection (ATCC). These cell lines were cultured in Dulbecco's minimum essential medium (DMEM, Gibco, Shanghai) mixed with $10 \%$ fetal bovine serum (FBS, Gibco, Shanghai) and 1\% antibiotics (streptomycin and penicillin, Gibco, Shanghai). The medium was replaced every $2-3$ days.

\section{Clinical tissues}

A total of 6 patients who were diagnosed with conventional osteosarcoma and underwent surgical resection at the Second Hospital of Jilin University were involved in our study. The diagnosis of OS was confirmed by pathological analysis. None of the patients received radiotherapy or chemotherapy before surgery. Osteosarcoma tissues (OST) and paired adjacent normal tissues were obtained after surgery and stored in liquid nitrogen. All the patients involved in this study provided written informed consents. The ethics committee of the Second Hospital of Jilin University approved this study (NO. 2016.169).

\section{RNA preparation and quantitative real-time PCR analysis}

Total RNA was extracted using TRIzol (Invitrogen) following the manufacturer's instructions. The cDNAs were synthesized using First-Strand Synthesis Kit (TAKARA, Tokyo, Japan). The primers of circRNA, miRNA and mRNA were synthesized by GenePharma (Suzhou, China). Quantitative real-time PCR (qRT-PCR) was performed using the TB Green ${ }^{\text {TM }}$ Kit (TAKARA, Tokyo, Japan). The expression of circRNA and mRNA were normalized relative to GAPDH, and miRNAs was normalized relative to U6, which was calculated using $2^{-\triangle \triangle \mathrm{Ct}}$ method. The primers used in this study are listed in Table 1.

\section{Luciferase reporter gene assay}

The 3'-UTR of PF4V1 and hsa_circ_0069117 containing the putative binding site (wide and mutated type) of miR-875-3p were inserted into pGL6-miR vectors which were then validated by sequencing (Genepharma, Suzhou, China). The $293 \mathrm{~T}$ cells were co-transfected by reporter vectors and miR-875-3p mimics (GenePharma, Suzhou, China). Firefly luciferase activity was measured at $48 \mathrm{~h}$ after transfection.

\section{Cell transfection}

The hsa_circ_0069117 overexpression vector (ovhsa_circ_0069117) and siRNA (si-hsa_circ_0069117) 
Table 1 Primers used in this study.

\begin{tabular}{lll}
\hline Gene name & Forward $\mathbf{( 5}^{\prime} \mathbf{-} \mathbf{3}^{\prime} \mathbf{)}$ & Reverse $\mathbf{( 5}^{\prime} \mathbf{- \mathbf { 3 } ^ { \prime } )}$ \\
\hline hsa_circ_0069117 & TGCGCATTTCAAGAAGAACA & TCCACTTCAGAGCCTCCTGT \\
miR-875-3p & CGCGCGCCTGGAAACACTGAG & ATCCAGTGCAGGGTCCGAGG \\
U6 & CGCTTCGGCAGCACATATAC & TTCACGAATTGGGTGTCATC \\
PF4V1 & GCCAGGAGATGCTGTTCTTG & GGGAGGTGGTCTTCACACAC \\
AKT & AGCGACGTGGCTATTGTGAAG & GCCATCATTCTTGAGGAGGAAGT \\
ERK1 & TACACCAACCTCTCGTACATCG & CATGTCTGAAGCGCAGTAAGATT \\
GAPDH & GCACCGTCAAGGCTGAGAAC & TGGTGAAGACGCCAGTGGA \\
\hline
\end{tabular}

were designed and purchased from Hanbio (Shanghai, China). The miR-875-3p inhibitor and mimics were purchased from GenePharma Gene (Suzhou, China). Lipofectamine $^{\mathrm{TM}} 3000$ Transfection Reagent (Invitrogen, Thermo Fisher Scientific Inc., Shanghai, China) was used as the transfection vehicle. The expression of hsa_circ_0069117, miR-875-3p and mRNAs was detected at $48 \mathrm{~h}$ post-transfection using quantitative real-time PCR.

\section{Western blot}

Proteins were separated by gel electrophoresis and transferred to a PVDF membrane. The PVDF membrane was incubated with primary antibodies against PF4V1, ERK1, p-ERK1, AKT, p-AKT, and GAPDH (Abcam, Shanghai, China) overnight at $4{ }^{\circ} \mathrm{C}$. Secondary antibodies (Abcam, Shanghai, China) were used to incubate the blots. The ECL luminescence reagent (Invitrogen, Thermo Fisher Scientific Inc., Shanghai, China) was then used to visualize the bands. The quantification analysis of protein was performed for PF4V1, ERK1, p-ERK1, AKT by normalizing to GAPDH.

\section{Cell proliferation}

The effect of hsa_circ_0069117 on MG63 and U2OS proliferation was evaluated using the cell counting kit-8 (CCK-8) assay. OS cells were seeded in 96-well plates and cultured to $80-90 \%$ density. CCK-8 solution was added to the cell culture plates at $24 \mathrm{~h}$ and $48 \mathrm{~h}$ (for $2 \mathrm{~h}$ ) after hsa circ_0069117 vector transfection. The optical density was measured at a wavelength of $450 \mathrm{~nm}$ to reflect the effect of hsa_circ_0069117 on MG63 and U2OS proliferation.

\section{Cell migration}

The effect of hsa_circ_0069117 on MG63 and U2OS cell migration was evaluated using a wound-healing assay. OS cells were seeded in 96-well plates and cultured to $80-90 \%$ density. A straight wound was created manually with a clean $100 \mu \mathrm{L}$ plastic pipette tip post hsa_circ_0069117 vector transfection. The cell migration areas were recorded by microscopy at $24 \mathrm{~h}$ and $48 \mathrm{~h}$ post hsa_circ_0069117 vector transfection.

\section{Data analysis}

The expression data were using the t-test to compare differences between two groups. One-way ANOVA was used to compare the differences among multiple groups and $p<0.05$ indicated that the difference was statistically significant. $|\mathrm{FC}| \geq 2$ and adjusted $p<0.05$ were set as the threshold for detecting dysregulated circRNAs and miRNAs. Bar graphs were constructed using GraphPad Prism 7.0. The ceRNA network was generated using Cytoscape software.

\section{Results}

CircRNA hsa_circ_0069117 was most markedly dysregulated in OS cells and predicted to competitively binding to miR-875-3p with PF4V1

The circRNA expression profile (GSE96964) and miRNA expression profile (GSE70367) were analyzed using GEO2R to detect the dysregulated circRNAs and miRNAs in OS. According to the criteria, eight upregulated and 102 downregulated circRNAs were screened out (Fig. 1A). A total of 58 upregulated and 126 downregulated miRNAs were detected in GSE70367 (Fig. 1B). Among these differentially expressed circRNAs, hsa circ_0069117 was the most markedly dysregulated in OS cells lines (fold change: $-10.24, p$ value: 0.00000881 ). Next, we predicted 60 potential target miRNAs of hsa circ_0069117 (Fig. 1C). However, among these targeted miRNAs, only miR-875-3p was dysregulated in OS with a fold change of 2.50 and a $p$ value of 0.0042548 . Venn analysis indicated that $30 \mathrm{mRNAs}$ were predicted to be miR-875-3p targets (Fig. 1D). The ceRNA network was constructed to show the potential relationship between hsa_circ_0069117, miR-875-3p, and target mRNAs (Fig. 1E). Then, we performed a literature review and found that PF4V1 has already been validated as miR875-3p target, which was also dysregulated in several tumor diseases [19]. 

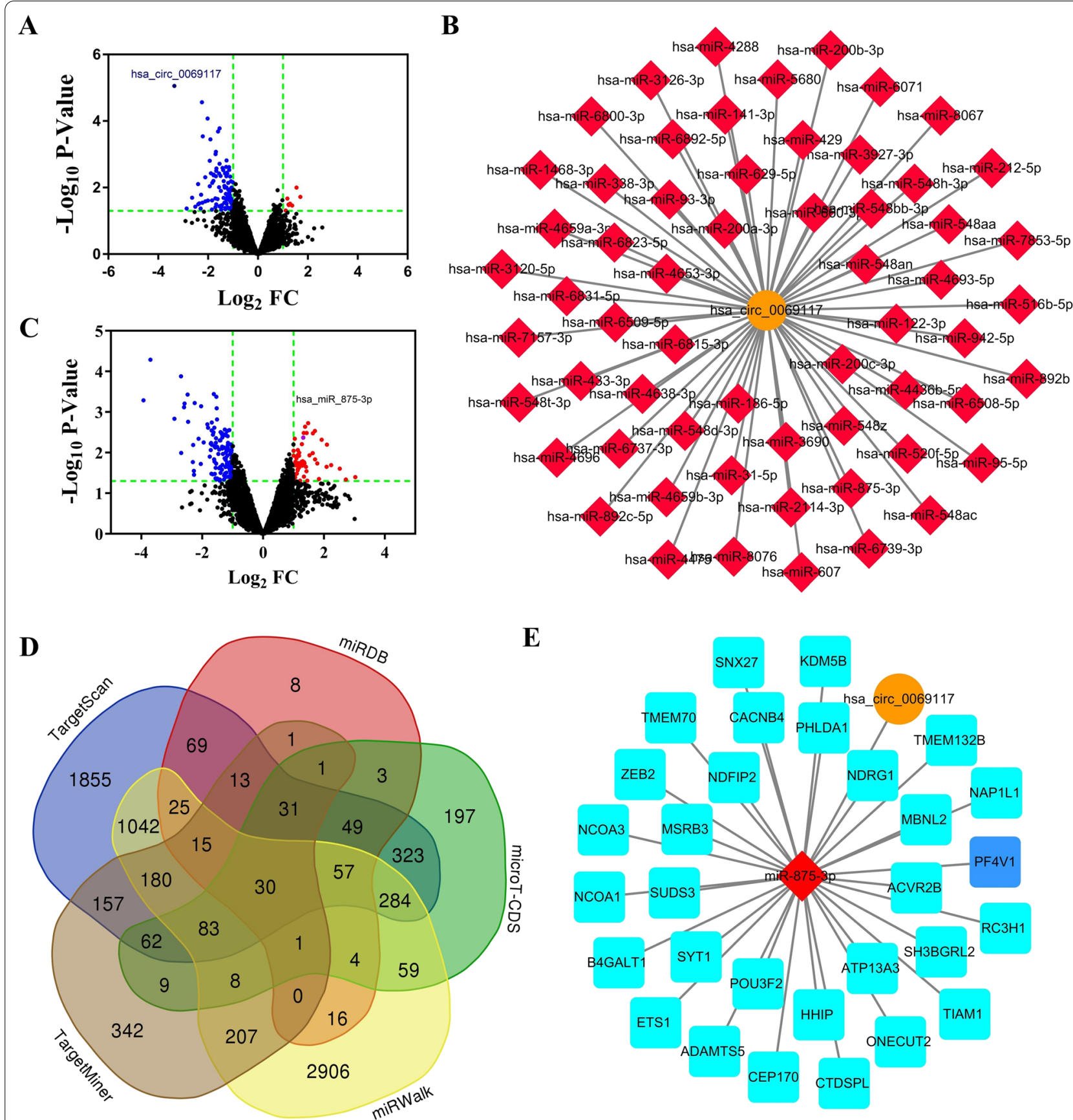

Fig. 1 A The volcano plots of circRNA expression profile. B The regulatory network of hsa_circ_0069117 and its potential target miRNAs. CThe volcano plots of miRNA expression profile. D Venn diagram showed there were 30 potential target mRNAs of miR-875-3p. EThe ceRNA networks showed the hsa_circ_0069117/miR-875-3p/mRNAs regulatory networks

The expression of hsa_circ_0069117, miR-875-3p, and PF4V1 was correlated in OS tissues

Next, we detected the expression of hsa_circ_0069117, miR-875-3p, and PF4V1 in four OSCLs. The results indicated that the expression of hsa_circ_0069117 was significantly decreased in OSCLs (Fig. 2A) while the expression of miR-875-3p was significantly increased in OSCLs (Fig. 2B), which was consistent with the results of circRNA (GSE96964) and miRNA (GSE70367) microarrays of OSCLs. The expression of PF4VA was significantly increased in OSCLs (Fig. 2C), which was consistent with the trend of hsa_circ_0069117. 


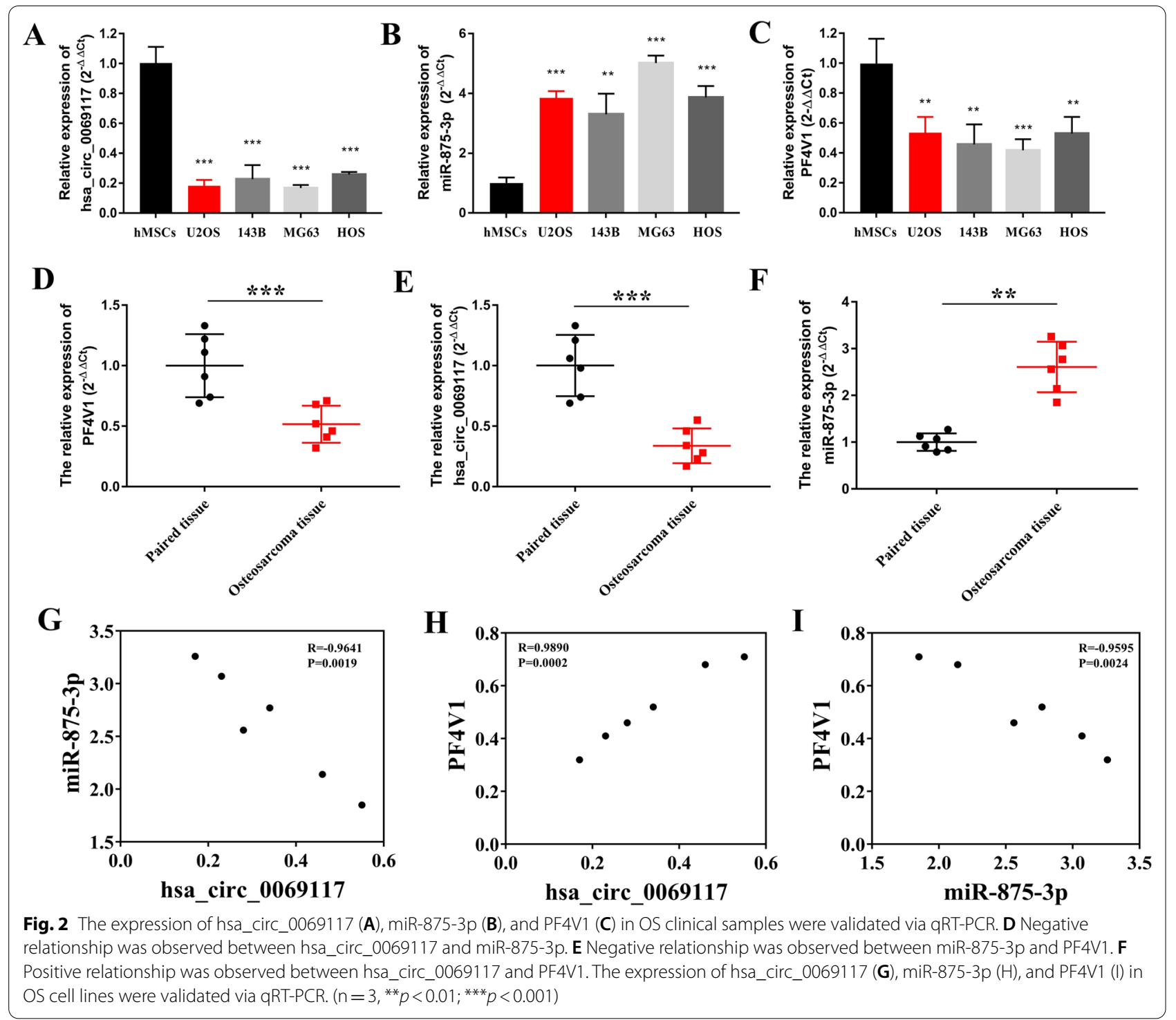

We further validated the expression of hsa circ_0069117/miR-875-3p/PF4V1 axis in OSTs, which was consistent with that in OSCLs (Fig. 2D-F). Interestingly, the expression of hsa_circ_0069117 was negatively correlated with that of miR-875-3p (Fig. 2G) and positively correlated with that of that of PF4V1 (Fig. 2H). Furthermore, a significant positive relationship was also observed between the expression of miR-875-3p and PF4V1 (Fig. 2I). These results suggest a regulatory relationship between hsa_circ_0069117, miR-875-3p, and PF4V1.

Thereafter, we explored the binding sites of miR-875-3p on hsa_circ_0069117 (Fig. 3A) and PF4V1 (Fig. 3B), which were further validated by luciferase reporter gene assays. The results showed that the luciferase activity of
hsa_circ_0069117-pGL6-miR and PF4V1-pGL6-miR vectors was significantly decreased when co-transfected with miR-875-3p mimics (Fig. 3C, D), while the other groups showed no significant changes.

CircRNA hsa_circ_0069117 could enhance the expression of PF4V1 by sponging miR-875-3p, thereby inhibiting the expression and phosphorylation of ERK1 and AKT Gain/loss-of-function assays were performed to verify the effect of hsa_circ_0069117 on miR-875-3p/PF4V1 expression and related pathways. The results showed that overexpressing hsa_circ_0069117 could significantly suppressed the expression of miR-875-3p (Fig. 4A). Inhibition of miR-875-3p by hsa_circ_0069117 promoted the expression of PF4V1 (Fig. 4A). Conversely, suppressing 


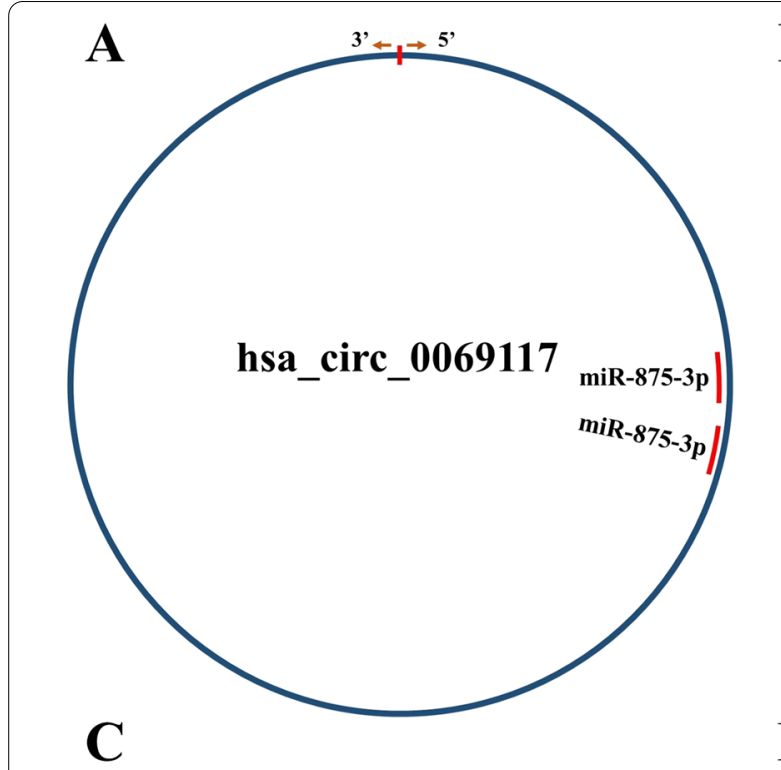

B

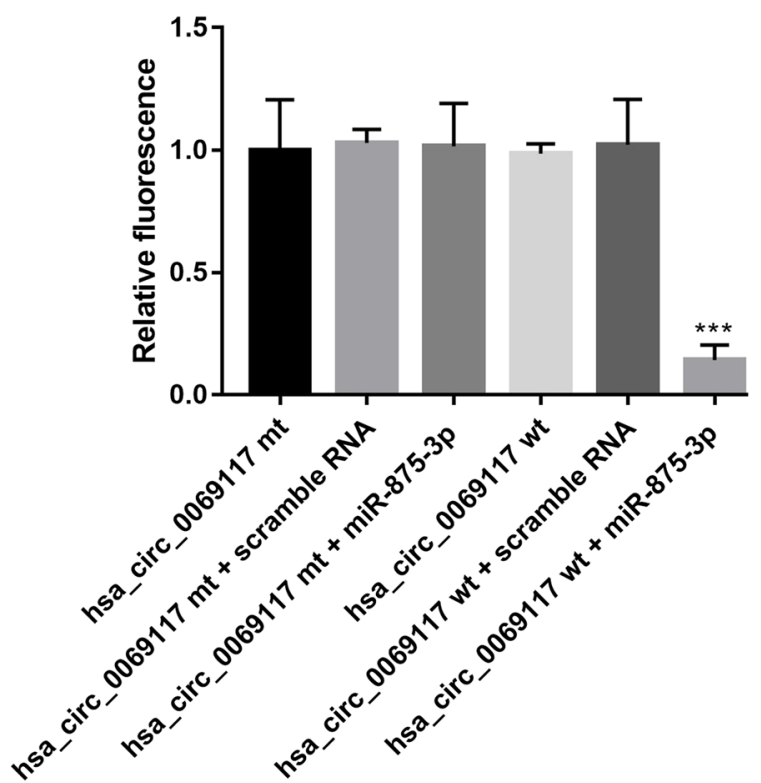

D

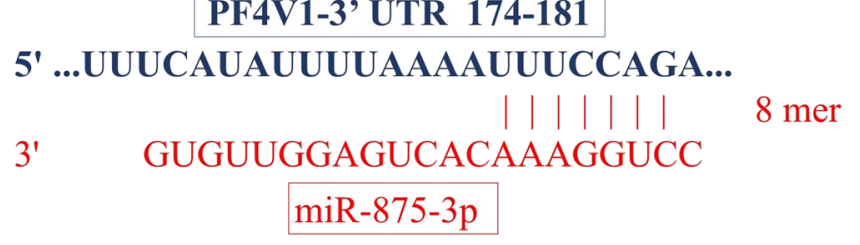

Fig. 3 A The binding position of miR-875-3p on hsa_circ_0069117. B The binding position of miR-875-3p on PF4V1. The luciferase reporter gene assay indicated that miR-875-3p mimics could decrease the fluorescent expression of hsa_circ_0069117-pGL6-miR (C) and PF4V1-pGL6-miR (D) vectors. $\left(n=3,{ }^{* * *} p<0.001\right)$

(See figure on next page.)

Fig. 4 A The expression trends of hsa_circ_0069117, miR-875-3p, and PF4V1after hsa_circ_0069117 overexpressing. B The expression trends of hsa_circ_0069117, miR-875-3p, and PF4V1 after hsa_circ_0069117 knocking-down. C Overexpressing hsa_circ_0069117 could promote the gene expression of PF4V1 but inhibit ERK1 and AKT, which could be reversed by co-transfecting miR-875-3p mimics. D and E Overexpressing hsa_circ_0069117 could promote the protein expression of PF4V1 but inhibit the protein expression and phosphorylation of ERK1 and AKT, which could also be reversed by co-transfecting miR-875-3p mimics. F Knocking-down hsa_circ_0069117 could inhibit the gene expression of PF4V1 but promote ERK1 and AKT, which could be reversed by co-transfecting miR-875-3p inhibitor. G and $\mathbf{H}$ Overexpressing hsa_circ_0069117 could promote the protein expression of PF4V1 but inhibit the protein expression and phosphorylation of ERK1 and AKT, which could also be reversed by co-transfecting miR-875-3p inhibitor. $\left(n=3,{ }^{* *} p<0.001\right)$ 


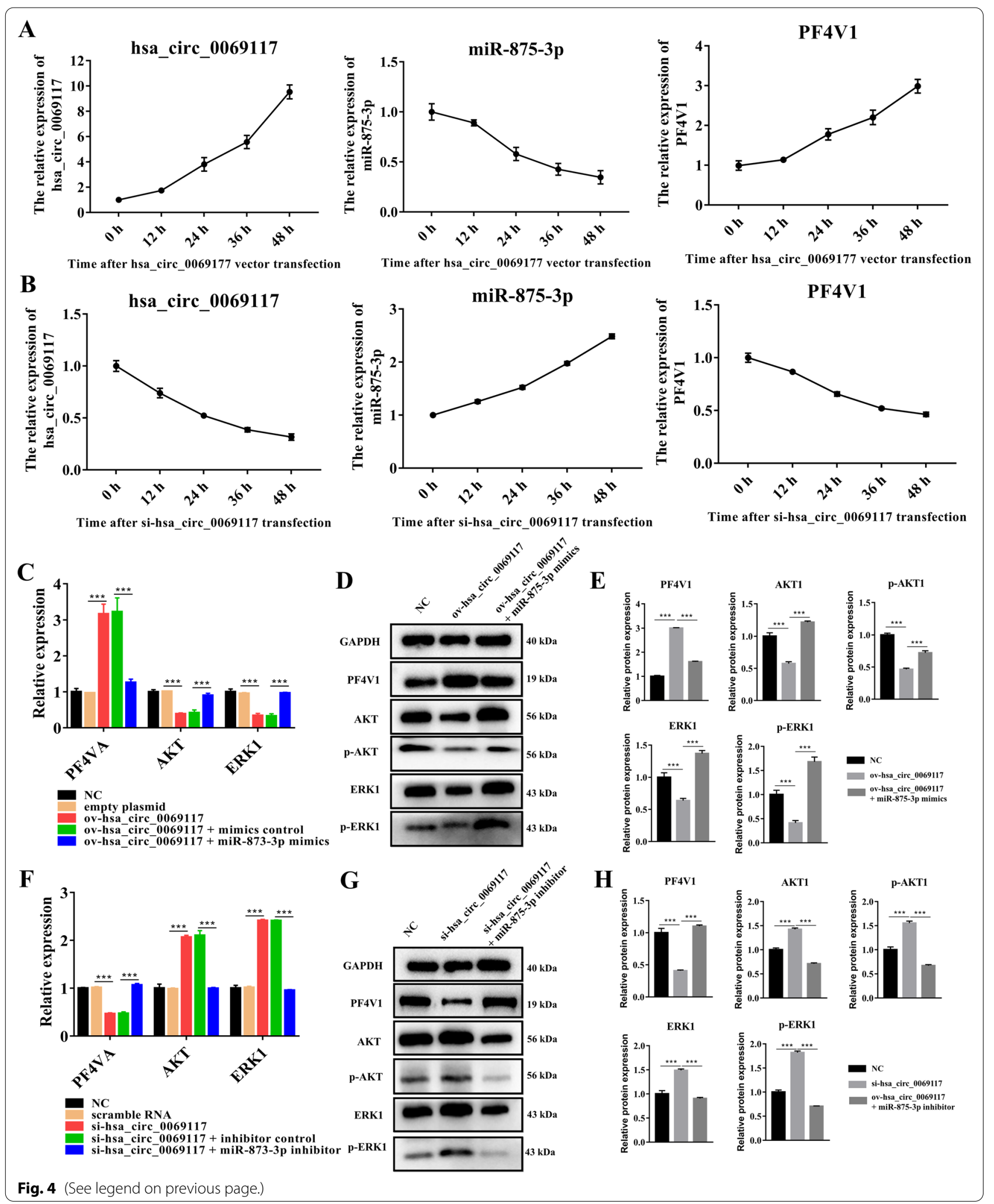


hsa_circ_0069117 significantly upregulated the expression of miR-875-3p thereby inhibiting the expression of PF4V1 (Fig. 4B).

ERK1 and AKT have been reported as downstream targets of PF4V1 [19]. Therefore, we further validated the effect of hsa_circ_0069117/miR-875-3p/PF4V1 on ERK1 and AKT expression. Overexpressing hsa_circ_0069117 significantly promoted the gene and protein expression of PF4V1 but suppressed the gene expression of ERK1 and AKT, while these effects could be reversed by overexpressing miR-875-3p as a rescue method (Fig. 4C). The expression and phosphorylation of ERK1 and AKT protein was consistent with the qRT-PCR results (Fig. 4D, E). Furthermore, knocking down hsa_circ_0069117 significantly inhibited the gene and protein expression of PF4V1 but promoted the gene expression of ERK1 and AKT, while these effects could be reversed by overexpressing miR-875-3p as a rescue method (Fig. 4F). The expression and phosphorylation of ERK1 and AKT protein was consistent with also the qRT-PCR results (Fig. 4G, H). The above results suggested that hsa_circ_0069117 could promote the expression of PF4V1 by sponging miR-875-3p thereby inhibiting the expression and phosphorylation of ERK1 and AKT.

\section{Overexpressing hsa_circ_0069117 suppresses the proliferation and migration of OS cells}

Subsequently, we validated the effect of hsa_circ_0069117 on the proliferation and migration of OS cells. As hsa circ_0069117 was most remarkably decreased in MG63 and U2OS cell lines, we performed CCK-8 and wound healing assays on MG63 and U2OS cell lines after hsa circ_0069117 overexpression. The results indicated that the proliferation (Fig. 5A) and migration (Fig. 5B, C) of MG63 and U2OS cell lines were attenuated as hsa circ_0069117 over-expressed. However, the attenuated effect of hsa_circ_0069117 on OS cell proliferation and migration could also be reversed by co-transfection with miR-875-3p mimics (Fig. 5B, C).

\section{Discussion}

Recent years have witnessed the great progression of circRNA research in functioning as a gene expression regulator in various diseases [20-22]. Several studies have revealed the dysregulation of circRNAs in OS, which affects many critical processes of OS, including proliferation, invasion, and metastasis [23-25]. Zhang et al. found that circ-0002052 could enhance the activation of Wnt/ $\mathrm{W}$-catenin and then stimulate the progression of OS via sponging miR-382 [23]. Yan et al. have reported that CircRNA PVT1 could promote metastasis via circPVT1/miR-526b/FOXC2 axis [26]. Yang et al. found circ_0001105 was expressed at low levels in OS cells, and their further study indicated it could suppress OS progression by regulating the miR-766/YTHDF2 axis [27]. Such preliminary studies indicated that different circRNAs could function as enhancers or suppressors of OS.

In present study, hsa_circ_0069117 was found to be the most markedly dysregulated in OS cells. However, the regulatory effect of hsa_circ_0069117 in malignant diseases, including OS, remains unclear. To further investigate the potential regulatory roles of hsa_circ_0069117 in OS, we predicted its potential target miRNAs. Sixty miRNAs were predicted to be targets of hsa_circ_0069117. By co-analyzing with the miRNA expression profile in OS, we found that only miR-875-3p was differentially expressed. MiRNA-875-3p has already been reported as an oncogene regulator by targeting PF4V1 [19]. PF4V1, also known as CXCL4L1, suppresses cell proliferation, migration, and invasion in various cancer by targeting ERK1 and AKT [28-30]. AKT/ERK pathway is critical intracellular signal transduction cascades, regulating cell proliferation and migration [31]. Previous studies indicated that AKT/ERK pathway could enhance the activity of osteosarcoma cell proliferation and migration and promoted osteosarcoma progression [32]. Based on these findings, we hypothesized that hsa_circ_0069117 could regulate the progress of OS via the miR-875-3p/PF4V1 axis (Fig. 6).

We constructed several lines to confirm our hypotheses [13]. First, we validated the expression of hsa circ_0069117, miR-875-3p, and PF4V1 in four OSCLs and OSTs. The results showed that the expression of hsa circ_0069117 and PF4V1 was decreased while the expression of miR-875-3p was increased, which was in line with our prediction. Second, we confirmed the binding sites of miR-875-3p on hsa_circ_0069117 and PF4V1 using luciferase reporter gene assay. Third, an in vitro functional verification assay of hsa_circ_0069117 was performed and the results showed that hsa_circ_0069117 could significantly suppress the expression of miR-875-3p and promote the expression of PF4V1. High level of PF4V1 could suppress the expression and phosphorylation of ERK1 and AKT [33, 34]. Fourth, the proliferation and migration of OSCLs could be attenuated by overexpressing hsa_circ_0069117 and reversed by co-transfection with miR-875-3p mimics.

In summary, we conducted this study to elucidate the regulatory effect of hsa_circ_0069117 on OS progression. We identified and further confirmed that miR-875-3p/ PF4V1 axis was the target of hsa_circ_0069117. Overexpression of hsa_circ_0069117 substantially attenuated the proliferation and migration of OS cells. Our study provides novel and reliable molecular target for the diagnosis and therapy of patients with OS. 


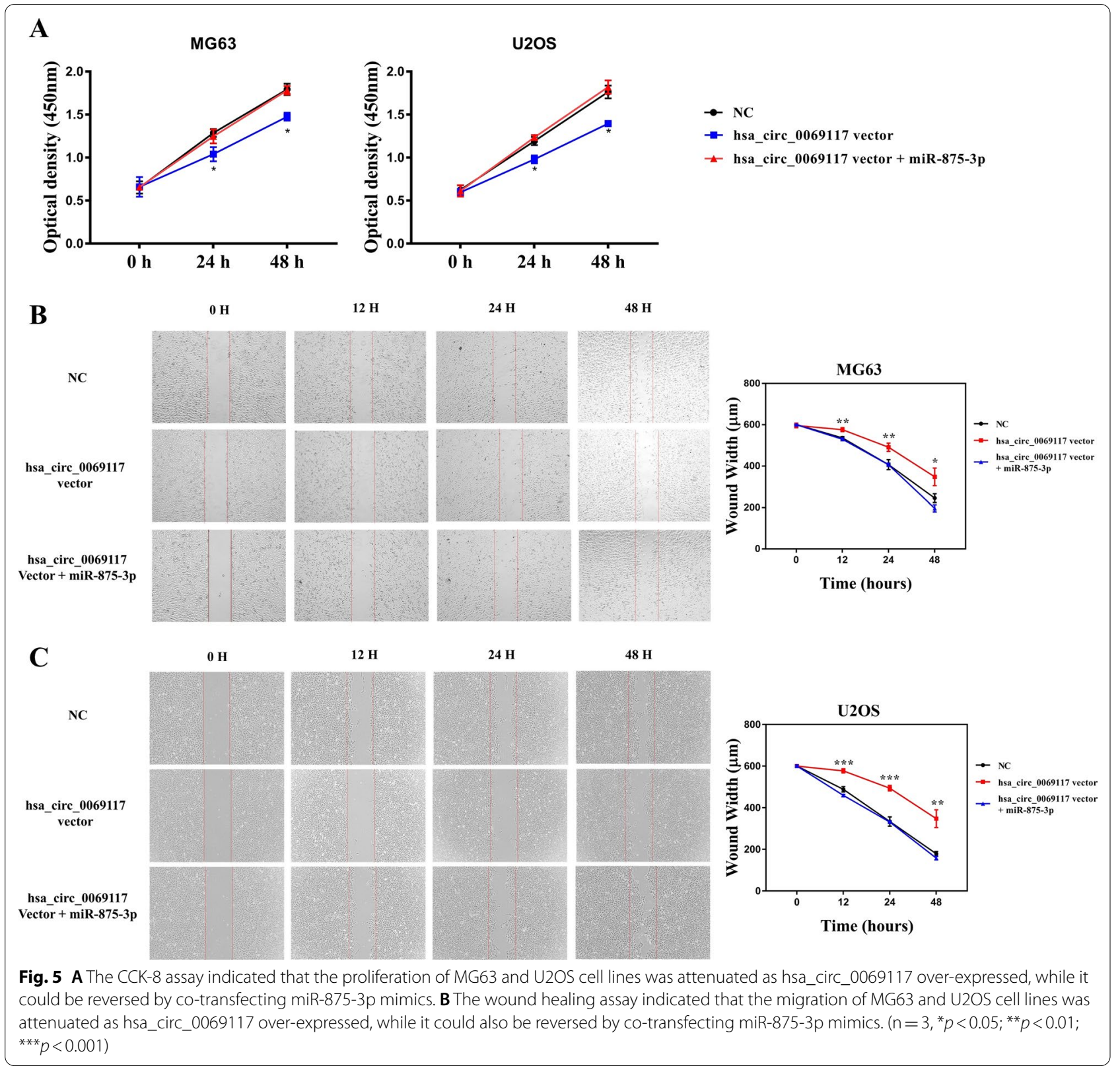

\section{Abbreviations}

AKT1: Protein kinase B; ATCC: American type culture collection; CCK8: Cell counting kit-8; circRNA: Circular RNA; ERK1: Extracellular signal-regulated kinase 1; FUNRICH: Functional enrichment analysis tool; GEO: Gene expression omnibus; ncRNA: Non-coding RNA; OS: Osteosarcoma; OSCL: Human osteosarcoma cell lines; OST: Osteosarcoma tissues; PF4V1: Platelet factor 4 variant 1; qRT-PCR: Quantitative real-time PCR; TBC1D14: TBC1 domain family member 14.

\section{Acknowledgements}

Not applicable.

\section{Authors' contributions}

$\mathrm{ZZ}$ and $\mathrm{XL}$ designed and supervised the study; ZZ and LZ performed the data analysis work; ZZ and SZ contributed to the verification work; ZZ and XL organized, designed, and wrote the paper. All authors read and approved the final manuscript.

\section{Funding}

Not applicable.

\section{Availability of data and materials}

All data generated during this study are available from the corresponding author. 


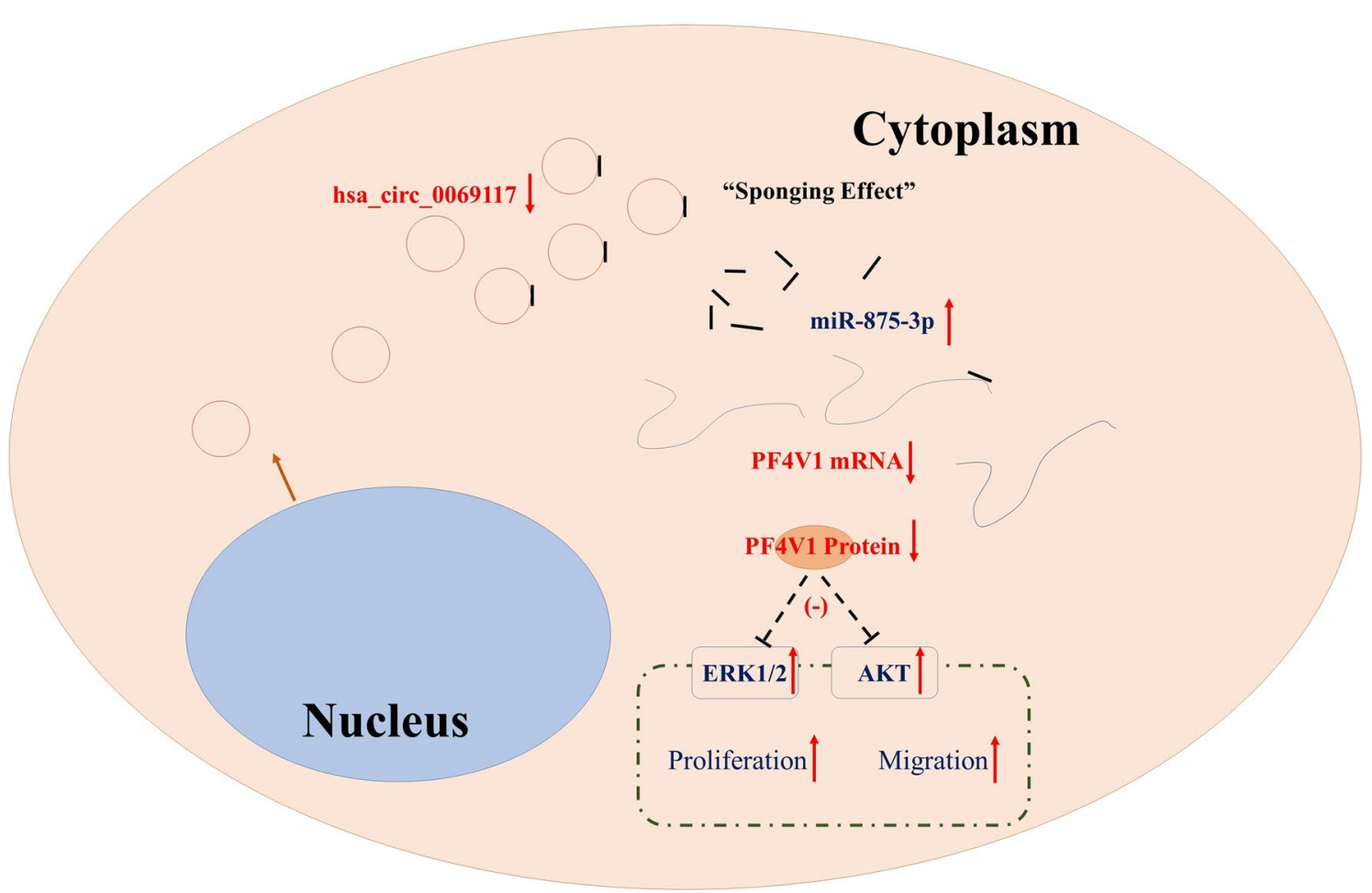

Fig. 6 The regulatory effect of hsa_circ_0069117 on OS cell proliferation and migration

\section{Declarations}

Ethics approval and consent to participate

The ethics committee of the Second Hospital of Jilin University approved this study.

\section{Consent for publication}

The authors consent for publication in the Journal.

\section{Competing interests}

The authors declare that they have no competing interests.

Received: 15 July 2021 Accepted: 6 January 2022

Published online: 21 January 2022

\section{References}

1. Moore DD, Luu HH. Osteosarcoma. Cancer Treat Res. 2014;162:65-92. https://doi.org/10.1007/978-3-319-07323-1_4.

2. Simpson E, Brown HL. Understanding osteosarcomas. JAAPA. 2018;31:159. https://doi.org/10.1097/01.JAA.0000541477.24116.8d.

3. Anderson ME. Update on survival in osteosarcoma. Orthop Clin North Am. 2016;47:283-92. https://doi.org/10.1016/j.ocl.2015.08.022.

4. Biazzo A, De Paolis M. Multidisciplinary approach to osteosarcoma. Acta Orthop Belg. 2016;82:690-8.

5. Kager L, Tamamyan G, Bielack S. Novel insights and therapeutic interventions for pediatric osteosarcoma. Future Oncol. 2017;13:357-68. https:// doi.org/10.2217/fon-2016-0261.

6. Harrison DJ, Geller DS, Gill JD, et al. Current and future therapeutic approaches for osteosarcoma. Expert Rev Anticancer Ther. 2018;18:39-50. https://doi.org/10.1080/14737140.2018.1413939.
7. Otoukesh B, Boddouhi B, Moghtadaei M, et al. Novel molecular insights and new therapeutic strategies in osteosarcoma. Cancer Cell Int. 2018;18:158. https://doi.org/10.1186/s12935-018-0654-4.

8. Li J, Sun D, Pu W, et al. Circular RNAs in cancer: biogenesis, function, and clinical significance. Trends Cancer. 2020;6:319-36. https://doi.org/10. 1016/j.trecan.2020.01.012.

9. Chen LL, Yang L. Regulation of circRNA biogenesis. RNA Biol. 2015;12:3818. https://doi.org/10.1080/15476286.2015.1020271

10. Guria A, Sharma P, Natesan S, et al. Circular RNAs-the road less traveled. Front Mol Biosci. 2019:6:146. https://doi.org/10.3389/fmolb.2019.00146.

11. Tran AM, Chalbatani GM, Berland L, et al. A new world of biomarkers and therapeutics for female reproductive system and breast cancers: circular RNAs. Front Cell Dev Biol. 2020;8:50. https://doi.org/10.3389/fcell.2020. 00050.

12. Kristensen LS, Andersen MS, Stagsted L, et al. The biogenesis, biology and characterization of circular RNAs. Nat Rev Genet. 2019;20:675-91. https:// doi.org/10.1038/s41576-019-0158-7.

13. Chen G, Wang Q, Li Z, et al. Circular RNA CDR1as promotes adipogenic and suppresses osteogenic differentiation of BMSCs in steroid-induced osteonecrosis of the femoral head. Bone. 2020;133: 115258. https://doi. org/10.1016/j.bone.2020.115258.

14. Wang C, Jing J, Cheng L. Emerging roles of non-coding RNAs in the pathogenesis, diagnosis and prognosis of osteosarcoma. Invest New Drugs. 2018;36:1116-32. https://doi.org/10.1007/s10637-018-0624-7.

15. Soghli N, Qujeq D, Yousefi T, et al. The regulatory functions of circular RNAs in osteosarcoma. Genomics. 2020. https://doi.org/10.1016/j.ygeno. 2020.03.024.

16. Chen G, Wang Q, Yang Q, et al. Circular RNAs hsa_circ_0032462, hsa_circ_0028173, hsa_circ_0005909 are predicted to promote CADM1 expression by functioning as miRNAs sponge in human osteosarcoma. PLOS ONE. 2018;13: e0202896. https://doi.org/10.1371/journal.pone. 0202896 
17. Chen $G, Y u W$, Li Z, et al. Potential regulatory effects of miR-182-3p in osteosarcoma via targeting EBF2. Biomed Res Int. 2019;2019:4897905. https://doi.org/10.1155/2019/4897905.

18. Pathan M, Keerthikumar S, Ang CS, et al. FunRich: an open access standalone functional enrichment and interaction network analysis tool. Proteomics. 2015;15:2597-601. https://doi.org/10.1002/pmic.201400515.

19. Li D, Hao X, Dong Y, et al. PF4V1, an miRNA-875-3p target, suppresses cell proliferation, migration, and invasion in prostate cancer and serves as a potential prognostic biomarker. Cancer Manag Res. 2019;11:2299-312. https://doi.org/10.2147/CMAR.S187831.

20. Hanniford D, Ulloa-Morales A, Karz A, et al. Epigenetic silencing of CDR1as Drives IGF2BP3-mediated melanoma invasion and metastasis. Cancer Cell. 2020;37:55-70.e15. https://doi.org/10.1016/j.ccell.2019.12.007.

21. Liu CX, Li X, Nan F, et al. Structure and degradation of circular RNAs regulate PKR activation in innate immunity. Cell. 2019;177:865-80.e21. https:// doi.org/10.1016/j.cell.2019.03.046.

22. Li Q, Wang Y, Wu S, et al. CircACC1 regulates assembly and activation of AMPK complex under metabolic stress. Cell Metab. 2019;30:157-73.e7. https://doi.org/10.1016/j.cmet.2019.05.009.

23. Zhang PR, Ren J, Wan JS, et al. Circular RNA hsa_circ_0002052 promotes osteosarcoma via modulating miR-382/STX6 axis. Hum Cell. 2020. https:// doi.org/10.1007/s13577-020-00335-9.

24. Xiong W, Zhang Y, Yu H. Comprehensive characterization of circular RNAs in osteosarcoma cell lines. Cell Signal. 2020;71: 109603. https://doi.org/10. 1016/j.cellsig.2020.109603.

25. Ji X, Shan L, Shen P, et al. Circular RNA circ_001621 promotes osteosarcoma cells proliferation and migration by sponging miR- 578 and regulating VEGF expression. Cell Death Dis. 2020;11:18. https://doi.org/10.1038/ s41419-019-2204-y.

26. Yan M, Gao H, Lv Z, et al. Circular RNA PVT1 promotes metastasis via regulating of miR-526b/FOXC2 signals in OS cells. J Cell Mol Med. 2020. https://doi.org/10.1111/jcmm.15215.

27. Yang J, Han Q, Li C, et al. Circular RNA circ_0001105 inhibits progression and metastasis of osteosarcoma by sponging miR-766 and activating YTHDF2 expression. Oncol Targets Ther. 2020;13:1723-36. https://doi.org/ 10.2147/OTT.S234668.

28. Odagiri $\mathrm{N}$, Matsubara T, Higuchi M, et al. Involvement of ERK1/2 activation in the gene expression of senescence-associated secretory factors in human hepatic stellate cells. Mol Cell Biochem. 2019;455:7-19. https:// doi.org/10.1007/s11010-018-3466-X.

29. RuytinX P, Proost P, Struyf S. CXCL4 and CXCL4L1 in cancer. Cytokine. 2018:109:65-71. https://doi.org/10.1016/j.cyto.2018.02.022.

30. Van Raemdonck K, Berghmans N, Vanheule V, et al. Angiostatic, tumor inflammatory and anti-tumor effects of CXCL4(47-70) and CXCL4L1 (47-70) in an EGF-dependent breast cancer model. Oncotarget. 2014:5:10916-33. https://doi.org/10.18632/oncotarget.2538.

31. Cao Z, Liao Q, Su M, et al. AKT and ERK dual inhibitors: the way forward. Cancer Lett. 2019;459:30-40. https://doi.org/10.1016/j.canlet.2019.05.025.

32. Tian K, Di R, Wang L. MicroRNA-23a enhances migration and invasion through PTEN in osteosarcoma. Cancer Gene Ther. 2015;22:351-9. https://doi.org/10.1038/cgt.2015.27.

33. Xu X, Yu H, XU Y. Ras-ERK1/2 Signaling promotes the development of osteosarcoma by regulating H2BK12ac through CBP [Expression of Concern]. Cancer Manag Res. 2020;12:2501. https://doi.org/10.2147/CMAR. S256769.

34. Wang S, Zhao G, Zhao S, et al. The effects of interleukin-33 (IL-33) on osteosarcoma cell viability, apoptosis, and epithelial-mesenchymal transition are mediated through the PI3K/AKT pathway. Med Sci Monit. 2020;26:e920766-71. https://doi.org/10.12659/MSM.920766.

\section{Publisher's Note}

Springer Nature remains neutral with regard to jurisdictional claims in published maps and institutional affiliations. 